\title{
Changes of Myelin Organization in Patients with Alzheimer's Disease Shown by q-Space Myelin Map Imaging
}

\author{
Miho Ota ${ }^{a}$ Noriko Sato $^{b}$ Yukio Kimura ${ }^{b}$ Yoko Shigemoto $^{b}$ \\ Hiroshi Kunugia Hiroshi Matsudac \\ ${ }^{a}$ Department of Mental Disorder Research, National Institute of Neuroscience, National \\ Center of Neurology and Psychiatry, Tokyo, Japan; b Department of Radiology, National \\ Center Hospital of Neurology and Psychiatry, Tokyo, Japan; ' Integrative Brain Imaging \\ Center, National Center of Neurology and Psychiatry, Tokyo, Japan
}

\section{Keywords}

Alzheimer's disease $\cdot$ Magnetic resonance imaging $\cdot$ Myelination $\cdot$ White matter

\begin{abstract}
Background: Recent studies detected the aberrant myelination of the central nervous system (CNS) in Alzheimer's disease (AD). Here, we compared the change of myelination between patients with $A D$ and controls by a novel magnetic resonance imaging modality, "q-space myelin map (MM) imaging." Methods: Twenty patients with $A D$ and 18 healthy subjects underwent $\mathrm{MM}$ imaging. We compared the MM metric between the 2 groups and examined the relationships between the metric and the clinical symptoms of $A D$. Results: $A D$ patients showed a significant reduction of MM metric in the hippocampus, insula, precuneus, and anterior cingulate regions. There was also a significant negative correlation between the duration of illness and the MM metric in the temporoparietal region. Conclusion: Our findings suggest that MM imaging could be a clinically proper modality to estimate the myelination changes in AD patients.

\section{Introduction}

Alzheimer's disease (AD) is the most common age-related neurodegenerative disease leading to dementia, demonstrating progressive declines in memory, language, and visuospatial abilities. Pathologically, AD is characterized by the accumulation of aggregated $\beta$-amyloid 1-42 (A $\beta 1-42$ ) and the assembly of neurofibrillary tangles of hyperphosphorylated 
Ota et al.: Changes of Myelin Organization in Patients with Alzheimer's Disease Shown by $\mathrm{q}$-Space Myelin Map Imaging

tau in the cerebral cortex, and AD is considered a mainly cortical dementia. The progression of AD pathology occurs several years before the development of dementia, and the myelin and oligodendrocytes are vulnerable to the insults including A $\beta$ and neurofibrillary tangles. These vulnerabilities are pertinent to $\mathrm{AD}$, and decreases of myelin density, axonal loss, loss of oligodendrocytes, astrogliosis, microglial activation, and dilated perivascular spaces in white matter were detected in an animal study [1], postmortem AD studies [2-4], and in vivo clinical AD studies using magnetic resonance methods $[5,6]$. A recent study focusing on preclinical $\mathrm{AD}$ also showed the relationship between $\mathrm{A} \beta$ amount and myelin water fraction [7].

A magnetic resonance imaging (MRI)-based evaluation of brain structures is regarded as a valid method to assess disease status and progression in AD [8], and several MRI-based studies have revealed AD-related changes in brain structures. Some meta-analyses focusing on the gray matter volume changes in $\mathrm{AD}$ detected volume decreases in areas such as the frontal gyrus, parietal gyrus, insula, hippocampus, cingulate gyrus, and precuneus [9-11]. Further, diffusion tensor imaging and magnetic transfer imaging (MTI) studies can quantify white matter microstructural changes which are believed to reflect mostly ischemic demyelination, axonal loss, and gliosis. Investigations using diffusion tensor imaging showed AD-related changes in temporal areas, parietal areas, frontal areas, the corpus callosum, cingulate gyrus/ cingulate fiber tracts, uncinate fasciculus, inferior longitudinal fasciculus/superior longitudinal fasciculus fiber pathways, hippocampus, thalamus, basal ganglia, and internal capsule (for a review, see [12]). A study using MTI revealed a decrease in the bilateral magnetization transfer ratio of $\mathrm{AD}$ patients in the temporal, cingulate, parietal, and prefrontal white matter regions, and these results indicated a widespread decrease of white matter myelination in $\mathrm{AD}$ [13]. Moreover, a study using the multi-echo T2 relaxation time technique, a valid marker of central nervous system (CNS) myelin density [14], also revealed the demyelination of AD in temporal/parietal and bilateral periventricular white matter regions [15]. Since then, myelin abnormalities in the CNS have been gaining increasing attention in AD research.

Myelin is a lipid-enriched structure that hinders the free diffusion of water molecules. The mean diffusivity and fractional anisotropy (FA) are comprehensible parameters of diffusion restriction. Thus, these parameters have been used to estimate the presence of the biological diffusion barrier myelin within the human CNS. However, it is based on assumption that the probability density function (PDF) of water diffusion is Gaussian in its shape. In reality, water diffusion is not only restricted by myelin, but also by cell membranes or by axonal sheaths, thereby producing non-Gaussianity in the PDF. q-Space diffusion MRI (QSI) enables the calculation of the PDF in a non-Gaussian fashion [16]. The key principle of QSI is that the PDF is derived from a Fourier transformation of a diffusion-related signal decay curve with respect to multiple b-values. The PDF obtained by QSI provides an ideal metric that can estimate the presence of myelin accurately. Diffusional kurtosis imaging (DKI) is based on the assumption that the PDF of water diffusion is non-Gaussian, and DKI can reduce the acquisition times by using a limited number of low b-values $\left(<2,000 \mathrm{~s} / \mathrm{mm}^{2}, 3\right.$ steps $)$. However, the DKI estimation equation is only valid for low $b$-values $\left(<3,500 \mathrm{~s} / \mathrm{mm}^{2}\right)$, but the diffusion-related signal decay curve in white matter is multiexponential and the accuracy of the PDF depends upon data with high b-values $\left(<10,000 \mathrm{~s} / \mathrm{mm}^{2}\right)$. As a result, diffusion resolution is sacrificed in DKI [17]. A study from 2016 by Fujiyoshi et al. [17] introduced a novel QSI modality that produces a "myelin map" (MM). To reduce the acquisition time, they applied high b-values while limiting their number $\left(<10,000 \mathrm{~s} / \mathrm{mm}^{2}, 9\right.$ steps). The MM accurately depicted the myelin status in mice and nonhuman primates and showed a significantly higher contrast in detecting myelin signals not only in deep white matter, such as the corpus callosum, but also in the cerebral cortex in the healthy human brain [17]. The MM also presented the demyelination and remyelination in a longitudinal clinical intervention study of patients with multiple sclerosis [18]. These results indicated that the MM, a kurtosis-related heat map 
Table 1. Demographic parameters of the participants
Ota et al.: Changes of Myelin Organization in Patients with Alzheimer's Disease Shown by $q$-Space Myelin Map Imaging

\begin{tabular}{lcc}
\hline & $\begin{array}{l}\text { Patients with AD } \\
(n=20)\end{array}$ & $\begin{array}{l}\text { Healthy subjects } \\
(n=18)\end{array}$ \\
\hline Age, years & $80.5 \pm 5.8$ & $71.3 \pm 5.3$ \\
Men/women & $5 / 15$ & $8 / 10$ \\
MMSE & $17.4 \pm 3.4$ & $28.8 \pm 1.5$ \\
Onset of disease, years & $77.0 \pm 6.7$ & \\
\hline
\end{tabular}

Values are means \pm standard deviations unless otherwise indicated. AD, Alzheimer's disease; MMSE, Mini-Mental State Examination.

obtainable with time-saving q-space imaging, may provide a clinically useful means of visualizing myelin in the human CNS.

Moreover, there have been several reports on the topic of diffusion MRI metrics and aging [19-21]. However, there have been no studies detecting the age-related brain changes by MM analysis. In this study, we first evaluated which age-related brain changes exactly occur in healthy subjects. Then, we compared, for the first time, the MM in the intracranial whole brain and the FA map between patients with AD and healthy controls. We also examined the possible relationship between the MM and symptoms of AD.

\section{Methods}

\section{Subjects}

First, to estimate the age-related changes of the MM, 18 healthy adult volunteers ( 5 males and 13 females; mean age $59.7 \pm 13.2$ years, range $33-78$ years) were recruited from the community through local magazine advertisements and our website announcement. Applicants were excluded if they had a medical history of CNS disease or severe head injury or if they met the criteria for substance abuse or dependence. Individuals who demonstrated a history of psychiatric illness or contact with psychiatric services were also excluded.

Next, 20 patients who had been diagnosed with probable AD according to the criteria of the National Institute on Aging-Alzheimer's Association were enrolled [22]. The characteristics of the participants are summarized in Table 1. All participants were tested with the Mini-Mental State Examination (MMSE) for cognitive impairment [23].

For the control group, 18 healthy adult volunteers were recruited from the community through local magazine advertisements and our website announcement. Applicants were excluded if they had a medical history of CNS disease or severe head injury or if they met the criteria for substance abuse or dependence. Individuals who demonstrated a history of psychiatric illness or contact with psychiatric services were also excluded.

Written informed consent for participation in the study was obtained from each patient and control group subject. This study was performed in accordance with the Declaration of Helsinki and was approved by the ethics committee of the National Center of Neurology and Psychiatry, Japan.

\section{MRI Data Acquisition and Processing}

MM imaging was performed on a 3-Tesla MR system (Siemens Medical Systems, Erlangen, Germany). High-spatial resolution, 3-dimensional (3D) T1-weighted images were used for the morphometric study. 3D T1-weighted images were acquired in the sagittal plane (repetition time [TR]/echo time [TE], 1,800/2.26; flip angle, $9^{\circ}$; effective section thickness, $0.8 \mathrm{~mm}$; slab thickness, $180 \mathrm{~mm}$; matrix, $320 \times 320$; field of view [FOV], $250 \times 250 \mathrm{~mm}$; number of 
Ota et al.: Changes of Myelin Organization in Patients with Alzheimer's Disease Shown by q-Space Myelin Map Imaging
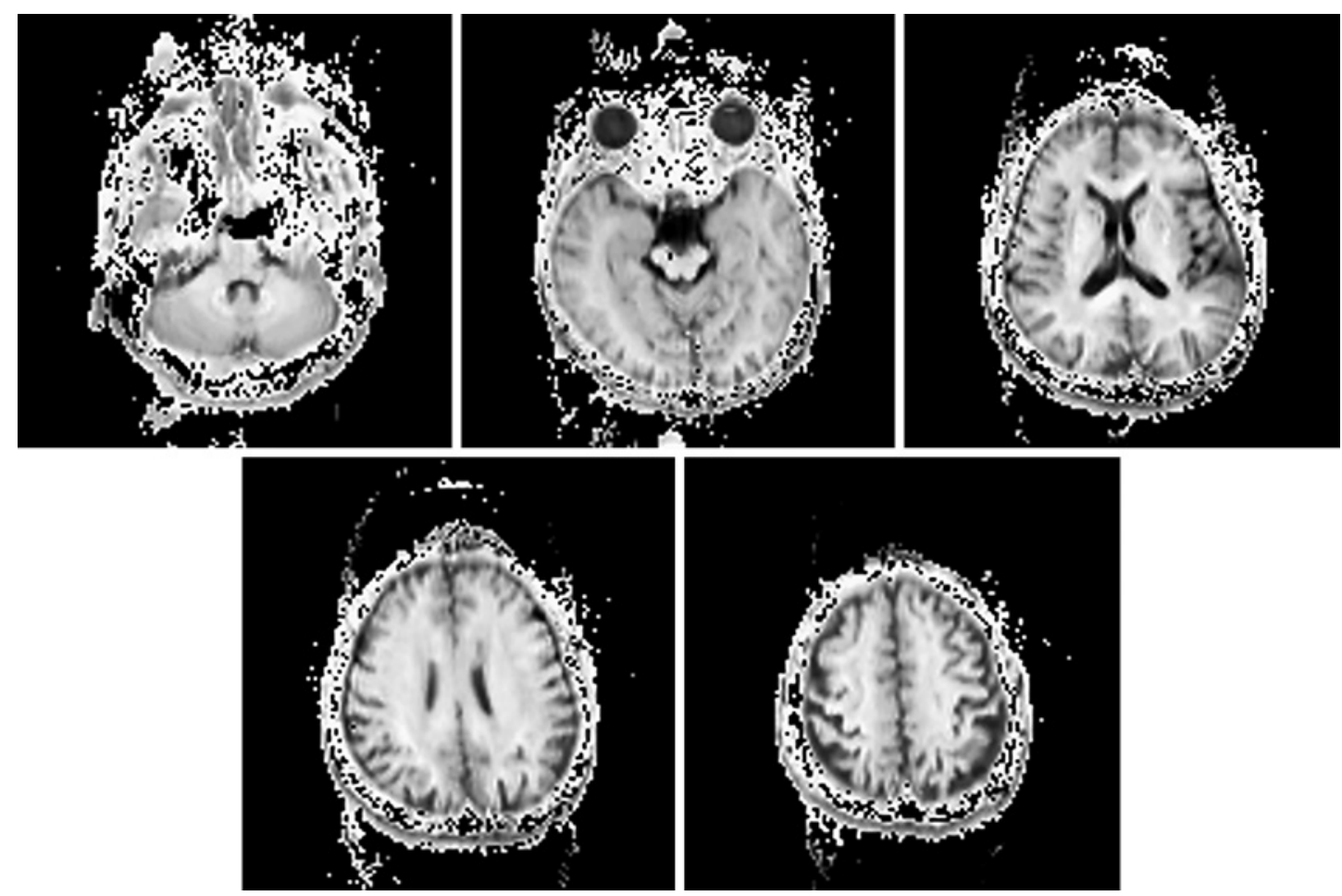

Fig. 1. Participant 1: a 55-year-old healthy woman. These images are the samples of the q-space myelin map imaging.

signals acquired, 1), yielding 224 contiguous slices through the brain. For the q-space MM imaging, the following parameters were used: TR/TE, 6,900/150; matrix, $128 \times 128$; FOV, $240 \times 240 \mathrm{~mm}$; slice thickness, $6 \mathrm{~mm}$; 9 b-values $(400,900,1,600,2,500,3,600,4,900,6,400$, 8,100 , and $10,000 \mathrm{~s} / \mathrm{mm}^{2}$ ) with 12 diffusion gradients and 2 images with no gradient $(\mathrm{A} \rightarrow \mathrm{P}$, $\mathrm{P} \rightarrow \mathrm{A}) ; 28$ interleaves. The q-space MM imaging examination took approximately $13 \mathrm{~min}$. The sample images are shown in Figure 1.

\section{Postprocessing of the Diffusional MRI Data}

Top-up and Eddy current-induced distortions of diffusion images were corrected using the Functional Magnetic Resonance Imaging of the Brain (FMRIB)'s Software Library (FSL) version 4.1 [24]. An in-house program (developed in IDL version 8.0; Exelis Visual Solutions, Broomfield, CO, USA) was used for further calculation and rendering of the MM [17]. To exclude the extraparenchymal noise, we masked the MMs with the binary mask image made by the skull-stripped intracranial images [25] derived from each individual 3D-T1 image. The FA maps were calculated from the raw data whose b-values were 900 by the FSL 4.1 software package [24].

\section{MRI Data Analysis}

For our evaluation of the MM metric and FA value of the intracranial whole brain by voxel-based morphometry (VBM) analysis, we normalized these images with the Diffeomorphic Anatomical Registration Through Exponentiated Lie (DARTEL) registration method [26]. First, each individual 3D-T1 image was co-registered and resliced to its $b=0$ image. Next, the co-registered 3D-T1 image was normalized with DARTEL. Finally, the transformation matrix was applied to the extraparenchymal noise-masked MMs and FA map. Each 
Fig. 2. Aging effect on the myelin map index. There were significant correlations between the age and myelin map index. The background images were "avg152T1" images regarded as the anatomical standard images in the SPM8. The same results were projected on the glass brain. R, right; L, left.

Ota et al.: Changes of Myelin Organization in Patients with Alzheimer's Disease Shown by $\mathrm{q}$-Space Myelin Map Imaging

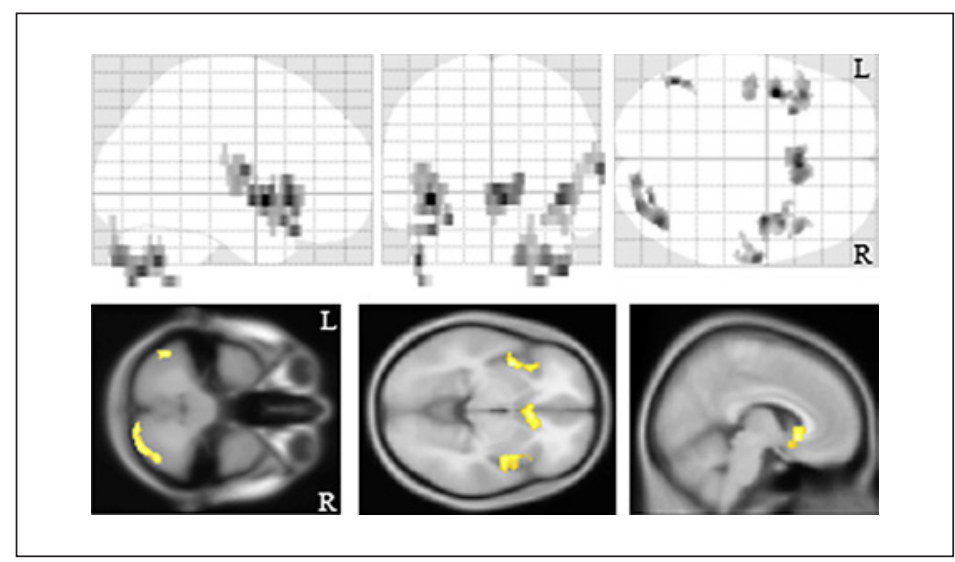

image was then smoothed with the use of a 6-mm full width at half maximum (FWHM) Gaussian kernel. For the gray matter volume, we preprocessed the 3D-T1 images using the VBM8 toolbox (http://dbm.neuro.uni-jena.de/vbm/), which is an extension of the unified segmentation model consisting of spatial normalization, bias field correction, and tissue segmentation [27]. Registration to the stereotactic space of the Montreal Neurological Institute consisted of linear affine transformation and nonlinear deformation using DARTEL normalization [18]. The bias-corrected, modulated, and warped tissue maps were smoothed with a 12-mm FWHM Gaussian kernel.

\section{Statistical Analyses}

We first evaluated the relationships between age and the index of MM and FA value by a multiple regression model in SPM8 using the subjects' gender as a nuisance variable. Only correlations that met these criteria were deemed significant. In this case, a seed level of $p$ value $<0.001$ (uncorrected) and a cluster level of $p<0.05$ was used (family-wise error [FWE]corrected).

Then, the regional myelin density and the gray matter volume differences between the patients with AD and the healthy subjects were evaluated by SPM8 using the subjects' age, education year, and gender as nuisance variables. The significance level was set at a seed level of $p$ value $<0.001$ (uncorrected) and a cluster level of $p<0.05$ (FWE-corrected). We also evaluated the relationships between the severity of AD evaluated by using the MMSE scores, the duration of illness, the regional myelination, and the gray matter volume in a multiple regression model in SPM8 using the subjects' age and gender as nuisance variables. Only correlations that met these criteria were deemed significant. In this case, the seed level of $p<0.001$ (uncorrected) and the seed level of $p<0.05$ (FWE-corrected) were selected.

\section{Results}

We estimated the aging effects on the index of MM. There were significant negative correlations between age and myelin density in the bilateral insulae, subgenual region, and cerebellum (Fig. 2).

Next, we compared the MM metric between the AD and control groups by a VBM analysis. The patients with $\mathrm{AD}$ showed a significant reduction of the MM metric in the left hippocampus, left insula, right precuneus, and bilateral anterior cingulate regions compared with healthy 


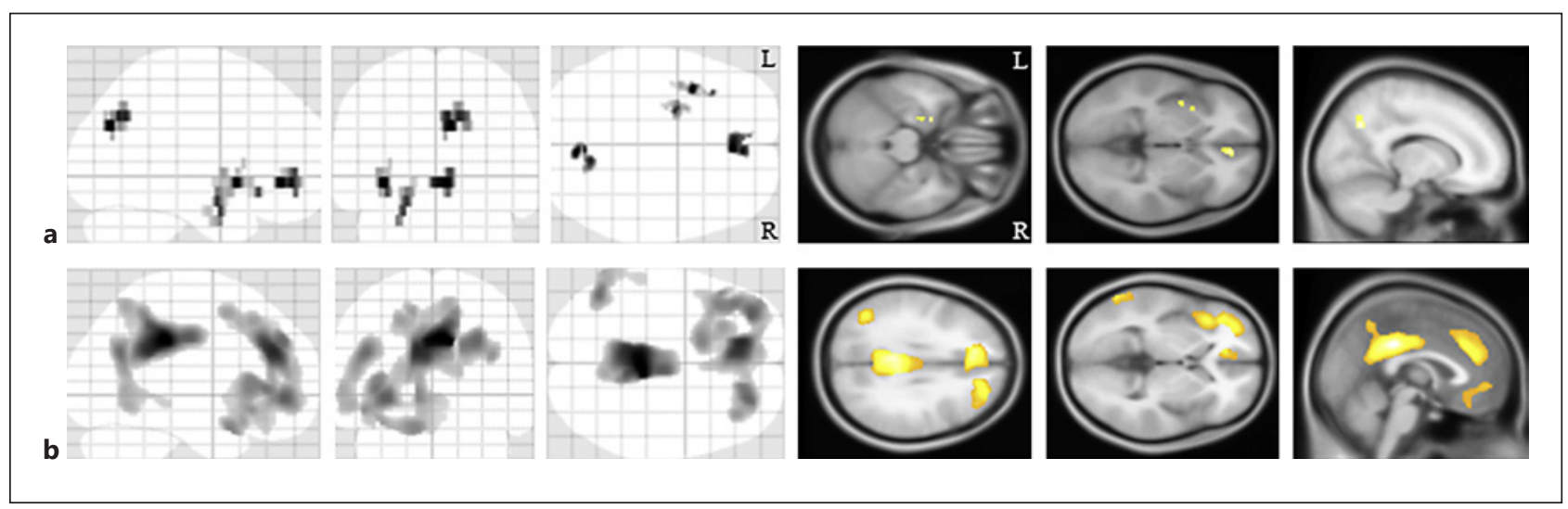

Fig. 3. The morphological brain changes in the patients with Alzheimer's disease. a Compared to the healthy subjects, the patients with Alzheimer's disease showed significant demyelination in the left hippocampus, left insula, bilateral anterior cingulate gyri, and the right precuneus. The same results were projected on the glass brain. $\mathbf{b}$ In the patients with Alzheimer's disease, there were significant gray matter volume decreases in the bilateral precuneus regions, anterior cingulate gyri, and prefrontal regions. The background images were "avg152T1" images regarded as the anatomical standard images in the SPM8. The same results were projected on the glass brain. R, right; L, left.

Fig. 4. The relationship between the myelination and the duration of illness in the patients with Alzheimer's disease. There was a significant negative correlation between the myelin density in the left temporoparietal regions of the patients with Alzheimer's disease and the duration of their illness. The background images were "avg152T1" images regarded as the anatomical standard images in the SPM8. The same results were projected on the glass brain. $\mathrm{R}$, right; L, left.

Table 2. Regions of significant decrease of myelin density in patients with Alzheimer's disease

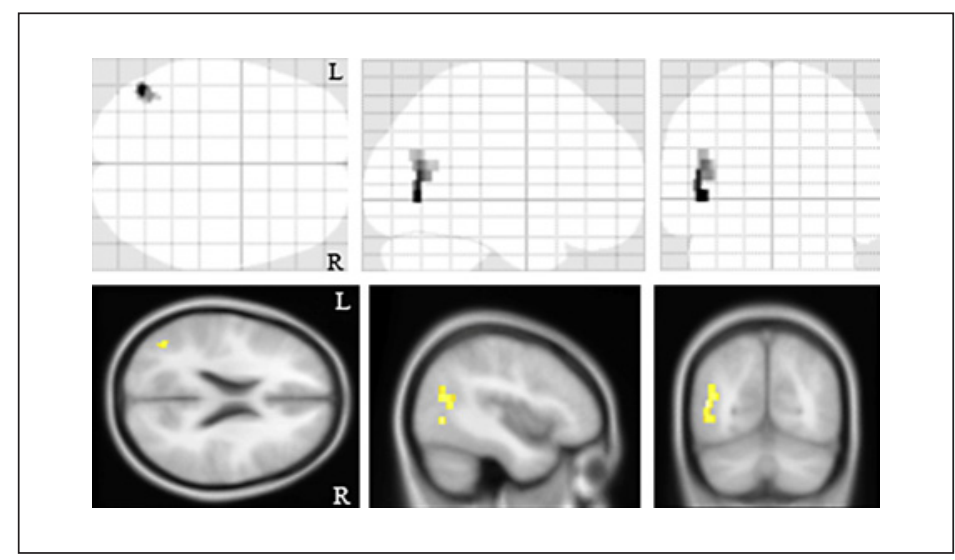

\begin{tabular}{lllrrl}
\hline $\begin{array}{l}\text { Cluster } \\
\text { size }\end{array}$ & $Z$-score & \multicolumn{1}{l}{$y$} & $Z$ & Brain region \\
\hline 51 & 4.11 & -36 & 9 & -6 & left insula \\
73 & 4.06 & 4 & 38 & -6 & right anterior cingulum \\
63 & 4.06 & 8 & -74 & 30 & right precuneus \\
52 & 3.8 & -21 & -4 & -18 & left hippocampus \\
\hline
\end{tabular}

subjects (Fig. 3a; Table 2). We also identified significant gray matter volume reductions in the $\mathrm{AD}$ patients in the bilateral precuneus regions, anterior cingulate gyri, prefrontal regions, and left temporal region (Fig. 3b). Regarding the FA value, there were no significant differences between the 2 groups. 
Ota et al.: Changes of Myelin Organization in Patients with Alzheimer's Disease Shown by $\mathrm{q}$-Space Myelin Map Imaging

We then evaluated the relationships between the clinical symptoms of the AD patients and the MM metrics and regional gray matter volume. There was a significant negative correlation between the duration of illness and the MM metric in the left temporoparietal region (cluster size, 72 voxels; $Z$-score, 4.32; [x,y, $]$, [-46, -68, 0]; Fig. 4), but we observed no other relationships between them. There were no significant relationships between the regional gray matter volumes and the clinical symptoms of AD.

\section{Discussion}

First, we found a significant negative relationship between the index of MM and age. Then, we identified significant reductions of myelin density in the left hippocampus, left insula, right precuneus, and bilateral anterior cingulate regions of the patients with $\mathrm{AD}$ compared to the healthy subjects. A significant correlation between the duration of illness and the myelin density in the left temporoparietal region was revealed by MM imaging. It is known that the accumulation of A 1-42 and hyperphosphorylated tau are the core pathology of $\mathrm{AD}$, and these give rise to the impairments of the myelin and oligodendrocytes in the early stage of the disease $[2,28,29]$. To our knowledge, however, the present study is the first study to detect the AD-related myelin damage revealed by q-space MM imaging.

In this study, we found age-related changes of myelin density in the insulae. It is well known that there are age-related changes of the insulae $[30,31]$. These points could be derived from the high sensitivity of the new metrics for microstructural changes.

Disease-related myelin damage is viewed in the context of the brain's continual homeostatic attempts to repair such damage [29]. The topography of myelination changes in AD is compatible with the distribution of amyloid plaques and that of neurofibrillary tangles [3234]. It is well known that individuals with $A D$ show changes in the frontal gyrus, parietal region, insula, hippocampus, cingulate gyrus, and precuneus [9-11], and our present findings are congruent with these points. Our VBM analysis with 3D-T1 images demonstrated the gray matter volume reductions of $\mathrm{AD}$ in the bilateral precuneus regions, cingulate gyri, prefrontal regions, and the left temporal region, and these lesions were larger than those revealed by q-space MM images. These differences might be derived from the difference in resolution between the 3D-T1 and MM images. Further investigations with high-resolution MM imaging could clarify this point.

Our findings revealed a significant correlation between the duration of illness and the myelin density in the left temporoparietal region shown by q-space MM images. Longitudinal VBM analyses focusing on AD have shown progressive cortical changes in the parietal region $[35,36]$, and our present results were compatible with those analyses. Because of the distribution of $\mathrm{AD}$ pathology, $\mathrm{AD}$ is typically considered a disease of the gray matter. However, the breakdown of myelin is associated with $\mathrm{AD}$ because the vulnerability of oligodendrocytes under Alzheimer's pathology enables the induction of myelin breakdown and loss of the myelin sheath, which might be the initiating step in the changes at the earliest stage of AD [3]. Here, by using q-space MM imaging, we were able to visualize the structural brain changes in $\mathrm{AD}$, and our findings can be said to be derived from the reaction to the progression of gray matter atrophy of $\mathrm{AD}$ and from the demyelination of white matter in the parietal region. We found this correlation using q-space MM images, but not using high-resolution 3D-T1 images. This point might indicate the effectiveness of myelin images for the subtle microstructural changes of the brain compared to the gray matter volume images. Preceding studies with MTI have calculated the white matter MM, but our present use of the novel MRI modality "q-space MM" enabled the estimation of the gray and white matter MMs simultaneously. This technique could thus be adopted hereafter as a mainstream technique in neuroimaging studies. 
Our present study has some limitations. First, the imaging resolution of MM was relatively low for the suppression of the inspection time. For that reason, we suspect that the protocol we used is unsuitable to estimate small regional disease-related changes. However, we were able to identify small changes such as those in the hippocampus, and our results may indicate that our MM protocol was suitable for the whole brain analysis. Additionally, our results showed the microstructural changes only in gray matter. Because of the distribution of its pathology and its associated neurodegeneration, AD is typically considered a disease of the brain's grey matter. Our results showed the disease-related changes in some gray matter regions, and these points were compatible with previous acknowledgements. However, our changes might result in, at least, the partial volume effects. Further studies of pre-mild cognitive impairment and preclinical AD patients showing less shrinkage of gray matter would clarify these points.

Second, the mean age of our AD subjects was higher than that of the healthy subjects. We controlled the effect of age in the statistical analyses, but further studies with age- and gendermatched AD patients and control subjects would shed light on this point.

\section{Conclusion}

We identified significant AD-related reductions of the MM index compared to healthy subjects, and our findings demonstrated a significant correlation between the clinical symptoms of $\mathrm{AD}$ and the regional MM metric as revealed by an MM whole brain voxel-based analysis. Our results are congruent with those of previous investigations, and we suggest that MM imaging is a clinically proper modality to estimate the myelinated changes in AD patients.

\section{Acknowledgement}

We thank Hata Junichi, $\mathrm{PhD}$, for his technical advice regarding the q-space myelin mapping.

\section{Statement of Ethics}

Written informed consent for participation in the study was obtained from each patient and control group subject. This study was performed in accordance with the Declaration of Helsinki and was approved by the ethics committee of the National Center of Neurology and Psychiatry, Japan.

\section{Disclosure Statement}

The authors have no conflicts of interest to report.

\section{Funding Sources}

This study was supported by the Brain Mapping by Integrated Neuro-Technologies for Disease Studies (Brain/MINDS) from the Japan Agency for Medical Research and Development (AMED) (\#17dm0207017h0004) to H.M. There were no other funding sources. 
Ota et al.: Changes of Myelin Organization in Patients with Alzheimer's Disease Shown by q-Space Myelin Map Imaging

\section{References}

1 Desai MK, Sudol KL, Janelsins MC, Mastrangelo MA, Frazer ME, Bowers WJ. Triple-transgenic Alzheimer's disease mice exhibit region-specific abnormalities in brain myelination patterns prior to appearance of amyloid and tau pathology. Glia. 2009 Jan;57(1):54-65.

2 Bartzokis G, Cummings JL, Sultzer D, Henderson VW, Nuechterlein KH, Mintz J. White matter structural integrity in healthy aging adults and patients with Alzheimer's disease: a magnetic resonance imaging study. Arch Neurol. 2003 Mar;60(3):393-8.

3 Cai Z, Xiao M. Oligodendrocytes and Alzheimer's disease. Int J Neurosci. 2016;126(2):97-104.

4 Shim YS, Yang DW, Roe CM, Coats MA, Benzinger TL, Xiong C, et al. Pathological correlates of white matter hyperintensities on magnetic resonance imaging. Dement Geriatr Cogn Disord. 2015;39(1-2):92-104.

5 Bartzokis G, Lu PH, Mintz J. Human brain myelination and amyloid beta deposition in Alzheimer's disease. Alzheimers Dement. 2007 Apr;3(2):122-5.

6 Bartzokis G, Lu PH, Mintz J. Quantifying age-related myelin breakdown with MRI: novel therapeutic targets for preventing cognitive decline and Alzheimer's disease. J Alzheimers Dis. 2004 Dec;6(6 Suppl):S53-9.

7 Dean DC 3rd, Hurley SA, Kecskemeti SR, O'Grady JP, Canda C, Davenport-Sis NJ, et al. Association of Amyloid Pathology With Myelin Alteration in Preclinical Alzheimer Disease. JAMA Neurol. 2017 Jan;74(1):41-9.

8 Frisoni GB, Fox NC, Jack CR Jr, Scheltens P, Thompson PM. The clinical use of structural MRI in Alzheimer disease. Nat Rev Neurol. 2010 Feb;6(2):67-77.

9 Chapleau M, Aldebert J, Montembeault M, Brambati SM. Atrophy in Alzheimer's disease and semantic dementia: an ALE meta-analysis of voxel-based morphometry studies. J Alzheimers Dis. 2016 Oct;54(3):941-55.

10 Wang WY, Yu JT, Liu Y, Yin RH, Wang HF, Wang J, et al. Voxel-based meta-analysis of grey matter changes in Alzheimer's disease. Transl Neurodegener. 2015 Mar; 4:6.

11 Yang J, Pan P, Song W, Huang R, Li J, Chen K, et al. Voxelwise meta-analysis of gray matter anomalies in Alzheimer's disease and mild cognitive impairment using anatomic likelihood estimation. J Neurol Sci. 2012 May;316(1-2):21-9.

12 Vasconcelos LG, Brucki SM, Jackowiski AP, Bueno OF. Diffusion tensor imaging for Alzheimer's disease: A review of concepts and potential clinical applicability. Dement Neuropsychol. 2009 Oct-Dec;3(4):268-74.

13 Fornari E, Maeder P, Meuli R, Ghika J, Knyazeva MG. Demyelination of superficial white matter in early Alzheimer's disease: a magnetization transfer imaging study. Neurobiol Aging. 2012 Feb;33(2):428.e7-19.

14 Laule C, Leung E, Lis DK, Traboulsee AL, Paty DW, MacKay AL, et al. Myelin water imaging in multiple sclerosis: quantitative correlations with histopathology. Mult Scler. 2006 Dec;12(6):747-53.

15 Kavroulakis E, Simos PG, Kalaitzakis G, Maris TG, Karageorgou D, Zaganas I, et al. Myelin content changes in probable Alzheimer's disease and mild cognitive impairment: associations with age and severity of neuropsychiatric impairment. J Magn Reson Imaging. 2018 May;47(5):1359-72.

16 King MD, Houseman J, Roussel SA, van Bruggen N, Williams SR, Gadian DG. q-Space imaging of the brain. Magn Reson Med. 1994 Dec;32(6):707-13.

17 Fujiyoshi K, Hikishima K, Nakahara J, Tsuji O, Hata J, Konomi T, et al. Application of q-space diffusion MRI for the visualization of white matter. J Neurosci. 2016 Mar;36(9):2796-808.

18 Tanikawa M, Nakahara J, Hata J, Suzuki S, Fujiyoshi K, Fujiwara H, et al. q-Space Myelin Map imaging for longitudinal analysis of demyelination and remyelination in multiple sclerosis patients treated with fingolimod: A preliminary study. J Neurol Sci. 2017 Feb;373:352-7.

19 Billiet T, Vandenbulcke M, Mädler B, Peeters R, Dhollander T, Zhang H, et al. Age-related microstructural differences quantified using myelin water imaging and advanced diffusion MRI. Neurobiol Aging. 2015 Jun;36(6): 2107-21.

20 Kodiweera C, Alexander AL, Harezlak J, McAllister TW, Wu YC. Age effects and sex differences in human brain white matter of young to middle-aged adults: A DTI, NODDI, and q-space study. Neuroimage. 2016 Mar; 128: 180-92.

21 Ota M, Sato N, Maikusa N, Sone D, Matsuda H, Kunugi H. Whole brain analyses of age-related microstructural changes quantified using different diffusional magnetic resonance imaging methods. Jpn J Radiol. 2017 Oct; 35(10):584-9.

22 McKhann GM, Knopman DS, Chertkow H, Hyman BT, Jack CR Jr, Kawas CH, et al. The diagnosis of dementia due to Alzheimer's disease: recommendations from the National Institute on Aging-Alzheimer's Association workgroups on diagnostic guidelines for Alzheimer's disease. Alzheimers Dement. 2011 May;7(3):263-9.

23 Folstein MF, Folstein SE, McHugh PR. "Mini-mental state". A practical method for grading the cognitive state of patients for the clinician. J Psychiatr Res. 1975 Nov;12(3):189-98.

24 Smith SM, Jenkinson M, Johansen-Berg H, Rueckert D, Nichols TE, Mackay CE, et al. Tract-based spatial statistics: voxelwise analysis of multi-subject diffusion data. Neuroimage. 2006 Jul;31(4):1487-505.

25 Iglesias JE, Liu CY, Thompson PM, Tu Z. Robust brain extraction across datasets and comparison with publicly available methods. IEEE Trans Med Imaging. 2011 Sep;30(9):1617-34.

26 Ashburner J. A fast diffeomorphic image registration algorithm. Neuroimage. 2007 0ct;38(1):95-113.

27 Ashburner J, Friston KJ. Unified segmentation. Neuroimage. 2005 Jul;26(3):839-51.

28 Bartzokis G. Age-related myelin breakdown: a developmental model of cognitive decline and Alzheimer's disease. Neurobiol Aging. 2004 Jan;25(1):5-18. 
29 Bartzokis G. Alzheimer's disease as homeostatic responses to age-related myelin breakdown. Neurobiol Aging. 2011 Aug;32(8):1341-71.

30 Bourisly AK, El-Beltagi A, Cherian J, Gejo G, Al-Jazzaf A, Ismail M. A voxel-based morphometric magnetic resonance imaging study of the brain detects age-related gray matter volume changes in healthy subjects of 21-45 years old. Neuroradiol J. 2015 Oct;28(5):450-9.

31 Good CD, Johnsrude IS, Ashburner J, Henson RN, Friston KJ, Frackowiak RS. A voxel-based morphometric study of ageing in 465 normal adult human brains. Neuroimage. 2001 Jul;14(1 Pt 1):21-36.

32 Frisoni GB, Lorenzi M, Caroli A, Kemppainen N, Någren K, Rinne JO. In vivo mapping of amyloid toxicity in Alzheimer disease. Neurology. 2009 Apr; 72(17):1504-11.

33 Nelson PT, Abner EL, Scheff SW, Schmitt FA, Kryscio RJ, Jicha GA, et al. Alzheimer's-type neuropathology in the precuneus is not increased relative to other areas of neocortex across a range of cognitive impairment. Neurosci Lett. 2009 Feb;450(3):336-9.

34 Shin J, Lee SY, Kim SH, Kim YB, Cho SJ. Multitracer PET imaging of amyloid plaques and neurofibrillary tangles in Alzheimer's disease. Neuroimage. 2008 Nov;43(2):236-44.

35 Landin-Romero R, Kumfor F, Leyton CE, Irish M, Hodges JR, Piguet O. Disease-specific patterns of cortical and subcortical degeneration in a longitudinal study of Alzheimer's disease and behavioural-variant frontotemporal dementia. Neuroimage. 2017 May;151:72-80.

36 Migliaccio R, Agosta F, Possin KL, Canu E, Filippi M, Rabinovici GD, et al. Mapping the progression of atrophy in early- and late-onset Alzheimer's disease. J Alzheimers Dis. 2015;46(2):351-64. 